\title{
Are Starburst Galaxies Proton Calorimeters?
}

\section{Xilu Wang* and Brian D. Fields}

University of Illinois at Urbana-Champaign, USA

E-mail: xwang107eillinois.edu, bdfieldseillinois.edu

In star-forming galaxies, gamma rays are mainly produced through the collision of high-energy protons in cosmic rays and protons in the interstellar medium (i.e., cosmic-ray induced $\pi^{0} \gamma$ radiation). For a "normal" star-forming galaxy like the Milky Way, most cosmic rays escape the galaxy before such collisions, but in starburst galaxies with dense gas and huge star formation rate, it is thought that most cosmic rays "die" in these interactions. To test this, we construct a "thicktarget" model for the starburst galaxies, in which cosmic rays are accelerated by supernovae, and escape is neglected. This model gives an upper limit to a galaxy's gamma-ray emission, and tests the calorimetric relation between gamma rays and cosmic rays for starbursts. Only two free parameters are involved in the model: the cosmic-ray proton acceleration energy per supernova $\varepsilon_{\mathrm{cr}}$, and the proton injection spectral index $s$. We apply our model to five observed starburst galaxies: M82, NGC 253, NGC 1068, NGC 4945 and the Circinus galaxy, and find most of these starbursts are consistent with being proton calorimeters, with $\varepsilon_{\mathrm{cr}} \sim(0.1-0.25) \times 10^{51} \mathrm{erg}$ and $s \sim 2.1-2.4$. But we confirm that for Circinus, other gamma-ray sources are needed to explain its $\mathrm{GeV}$ luminosity.

The 34th International Cosmic Ray Conference,

30 July- 6 August, 2015

The Hague, The Netherlands

\footnotetext{
* Speaker.
} 


\section{Introduction}

Cosmic rays (CRs) are accelerated by supernova explosions (e.g., $[1,2,3])$, and thus cosmic rays should be present in all galaxies hosting supernovae. As CRs propagate in the interstellar medium (ISM), inelastic collisions between CR and interstellar nuclei-both dominantly protonsyield pionic gamma rays via $p_{\mathrm{cr}} p_{\text {ism }} \rightarrow p p \pi^{0}, \pi^{0} \rightarrow \gamma \gamma[4,5]$. This hadronic process occurs in the Milky Way, but should also in other star-forming galaxies (e.g., [6, 7, 8, 9, 10, 11]). Fermi-LAT is the first $\mathrm{GeV}$ gamma-ray telescope to study external star-forming galaxies as a population. Fermi has detected three ordinary external star-forming galaxies: the Large Magellanic Cloud (LMC [12]), the Small Magellanic Cloud (SMC [13]) and M31 [14]. Fermi also detects five starburst galaxies: M82 and NGC253 [15], NGC4945 and NGC1068 [16], as well as the Circinus galaxy [17]. The two nearest and brightest starbursts, M82 and NGC253, are also detected at TeV energies by VERITAS [18] and H.E.S.S. [19, 20], respectively.

Starburst galaxies are a particularly interesting class of star-forming galaxies. Compared with normal galaxies like Milky Way, starbursts have exceptionally high star-formation rates and harbor regions of very dense gas. Thus cosmic rays accelerated in starbursts are expected to "die" in collisions with ISM rather than escape. In the limit where all the CR nuclei interact with ISM, a large fraction of proton energy is emitted as gamma rays, making such a galaxy a "proton calorimeter" (e.g., $[21,22,20]$ ). This situation has the maximum efficiency to convert supernova blast energy into gamma rays.

Our aim is to self-consistently calculate the pionic emission from starbursts in a closed box, and to use starburst data to test this calorimetric scenario. Existing models for nonthermal particles in starbursts have included both hadronic and leptonic processes such as synchrotron radiation, inverse Compton scattering and pion production (e.g., [23, 24, 22, 25]). Many-but not all-of these models predict that hadronic processes dominate above a few $\mathrm{GeV}$. By construction, our more focused model is economical and thus easy to test: it contains only two parameters, the cosmic-ray acceleration energy per supernova $\varepsilon_{\mathrm{cr}}$, and the cosmic-ray injection index $s$. The next section will briefly introduce the model. Results are presented in section 3. In section 4, further discussions and conclusions are given. For detailed model construction and results, see [26].

\section{Theoretical model: the Thick-Target/Calorimetric Model}

Our model is a thick-target "closed-box" model which has the following basic assumptions: 1) cosmic-rays and ISM particles distributions are spatially homogeneous (one zone); 2) cosmic-rays are initially accelerated by supernovae $(\mathrm{SN})$ with acceleration energy per $\mathrm{SN} \varepsilon_{\mathrm{cr}} ; 3$ ) the injected cosmic-ray/proton spectrum is a power law in momentum, of spectral index $s$ in $\mathrm{GeV}$ and $\mathrm{TeV}$ energy range; 4) all the cosmic rays will interact with ISM, i.e., the escape rate of protons is zero; 5) among the gamma-ray production mechanisms, pion production and decay dominates.

We adopt an injected cosmic-ray spectrum is $q_{\mathrm{p}} \equiv d q / d p=d N_{\mathrm{p}} / d V d t d E \propto p^{-s}$, with $p$ is the proton momentum, and the free parameter $s>2$ is the proton injection index. Thus the cosmic ray injected power, i.e., luminosity $d E_{\mathrm{cr}} / d t=L_{\mathrm{cr}}=\int E q_{p} d p d V$. Let cosmic rays be accelerated by supernovae that explode at a rate $R_{\mathrm{sn}}$. Then the normalization of $q_{\mathrm{p}}$ is fixed via energy conservation: 
$L_{\mathrm{cr}}=\varepsilon_{\mathrm{cr}} R_{\mathrm{sn}}$, which gives $q_{p} \propto \varepsilon_{\mathrm{cr}} R_{\mathrm{sn}} p^{-s}$. Here $\varepsilon_{\mathrm{cr}}$ is the mean energy per SN going into CRs. This is our other free parameter.

We describe cosmic-ray propagation (e.g., [27]) with a one-zone, closed-box, equilibrium model. Losses are only due to cosmic-ray interactions with ISM, and omit escape. Considering the elastic [28] and ionic energy loss rate [2], energy loss (per nucleon) per time is $b\left(n_{\mathrm{gas}}, E_{\mathrm{p}}\right)=$ $-d E / d t=b_{\text {inelastic }}+b_{\text {elastic }}+b_{\text {ionic }}, E_{\mathrm{p}}$ is the total energy of proton, $n_{\text {gas }}$ is the atomic hydrogen density of ISM. This fixes the calorimetric propagated proton flux to be:

$$
\phi_{\mathrm{p}}(E)=\frac{d N_{\mathrm{p}}}{d A d t d E}=\frac{v_{\mathrm{p}}}{b\left(n_{\mathrm{gas}}, E_{\mathrm{p}}\right)} \int_{E_{\mathrm{p}}}^{\infty} q_{\mathrm{p}}(E) d E \propto \varepsilon_{\mathrm{cr}} R_{\mathrm{sn}} I\left(>E_{\mathrm{p}}, s\right)
$$

where $v_{\mathrm{p}}$ is the velocity of proton, $I$ is here a function of $E_{\mathrm{p}}$ and $s$.

Adopting ref. [5]'s approach in getting $\pi^{0}$ productions' differential cross-section, we can calculate $b_{\text {inelastic }}$ self-consistently. And using the pion spectrum $q_{\pi}$ and gamma-ray spectrum $q_{\gamma}$ equations, our model's gamma-ray number flux from a starburst galaxy is:

$$
F_{\gamma}=\frac{d N_{\gamma, \mathrm{observed}}}{d E_{\gamma} d A d t}=\mathscr{A} \cdot \frac{1}{4 \pi d^{2}} \cdot \frac{d N_{\gamma}\left(E_{\gamma} ; \varepsilon_{\mathrm{cr}}, s\right)}{d E_{\gamma}} \cdot R_{\mathrm{sn}}
$$

where $\mathrm{d}$ is the distance of the galaxy, $E_{\gamma}$ is the total energy of photon. The gamma-ray flux is an observable from Fermi. $N_{\gamma \text {,observed }}$ is the gamma-ray number measured by the telescope. The galaxy's "effective" gamma-ray number yield per supernova $N_{\gamma}$ (gamma-rays are indirectly created by supernovae) is calculated in our model $\left(N_{\gamma} \propto \varepsilon_{\mathrm{cr}}\right)$. The total gamma ray production rate is the production of $N_{\gamma}$ and $R_{\mathrm{sn}}$. To account for the contribution from particle interactions involving nuclei with atomic weights $A>1$ in both CRs and ISM, a nuclear enhancement factor of $\mathscr{A}=0.59$ is included in the calculation [26].

We can see that, for a certain starburst galaxy, our model's gamma-radiation results only depend on two parameters: cosmic-ray proton acceleration energy per supernova $\varepsilon_{\mathrm{cr}}$ (direct proportionality) and the injected proton spectral index s. So we only need to vary the two parameters $\varepsilon_{\mathrm{cr}}$ and $\mathrm{s}$ to find the best fit of the model to observed data by Chi-squared test.

\section{Results}

\subsection{Individual Starbursts}

We apply our thick-target, closed-box model to five starbursts with GeV data. For each galaxy we adopt/infer an observed star-formation rate (SFR), and then calculate the pionic flux $E_{\gamma}^{2} d N_{\pi \rightarrow \gamma \gamma} / d E_{\gamma} d A d t$ as in $\left(\varepsilon_{\mathrm{cr}}, s\right)$ space. We perform $\chi^{2}$ test with the observed gamma-ray data to get the best-fit model parameters, which appear in Table 1, with $\varepsilon_{\text {cr }}$ in units of 1 foe $\equiv 10^{51} \mathrm{erg} \equiv$ 1Bethe.

We plot the best-fit pionic gamma-ray spectra of NGC253 and Circinus in Figure 1. These cases bracket the extremes, and illustrate general features of our model spectra: (1) the shape only depends on the injected proton spectrum; (2) the magnitude is proportional to $\varepsilon_{\mathrm{cr}}$; (3) at high energies, the gamma-ray spectral index is the same as the proton injection index $s$, and is flatter than in the escape-dominated galaxies; (4) the peak energy is insensitive to $s$, and corresponds to the "pion-bump" feature [4, 5]. For NGC253, we see that our best fit to $\mathrm{GeV}$ and $\mathrm{TeV}$ data is quite 


\begin{tabular}{c|c|ccc|cc}
\hline \hline $\begin{array}{c}\text { Galaxy } \\
\text { Name }\end{array}$ & $\begin{array}{c}\text { SN Rate } R_{\text {SN }} \\
\text { century }^{-1} \text { ] }\end{array}$ & $\begin{array}{c}\text { CR source } \\
\text { index } \hat{s}\end{array}$ & $\begin{array}{c}\text { CR acceleration energy } \\
\text { per SN } \varepsilon_{\text {cr }}[\text { foe } / S N]\end{array}$ & $\Gamma^{\text {Fermi }}$ & $\begin{array}{c}\text { GeV data } \\
\text { reference }\end{array}$ & $\begin{array}{c}\text { TeV data } \\
\text { reference }\end{array}$ \\
\hline M82 & $5.7 \pm 0.9$ & $2.275 \pm 0.102$ & $0.106 \pm 0.025$ & $2.25 \pm 0.13[29]$ & {$[18]$} & \\
NGC253 & $2.6 \pm 0.4$ & $2.350 \pm 0.037$ & $0.116 \pm 0.013$ & $2.18 \pm 0.09$ & {$[30]$} & {$[20]$} \\
NGC4945 & $3.2 \pm 0.9$ & $2.400 \pm 0.446$ & $0.210(>0.103)$ & $2.05 \pm 0.13$ & {$[29]$} & \\
NGC1068 & $35 \pm 9$ & $2.100 \pm 0.617$ & $0.253(>0.128)$ & $2.29 \pm 0.19$ & {$[29]$} & {$[31]$} \\
Circinus & $1.9 \pm 0.5$ & $2.300 \pm 0.486$ & $0.619(>0.310)$ & $2.19 \pm 0.12$ & {$[17]$} & \\
\hline \hline
\end{tabular}

Table 1: Results and parameters set for the starbursts in the "Thick-Target" Model. Fermi power-law fits for the gamma data of starbursts are from ref. [17] with $d N / d E \propto E^{-\Gamma}$.
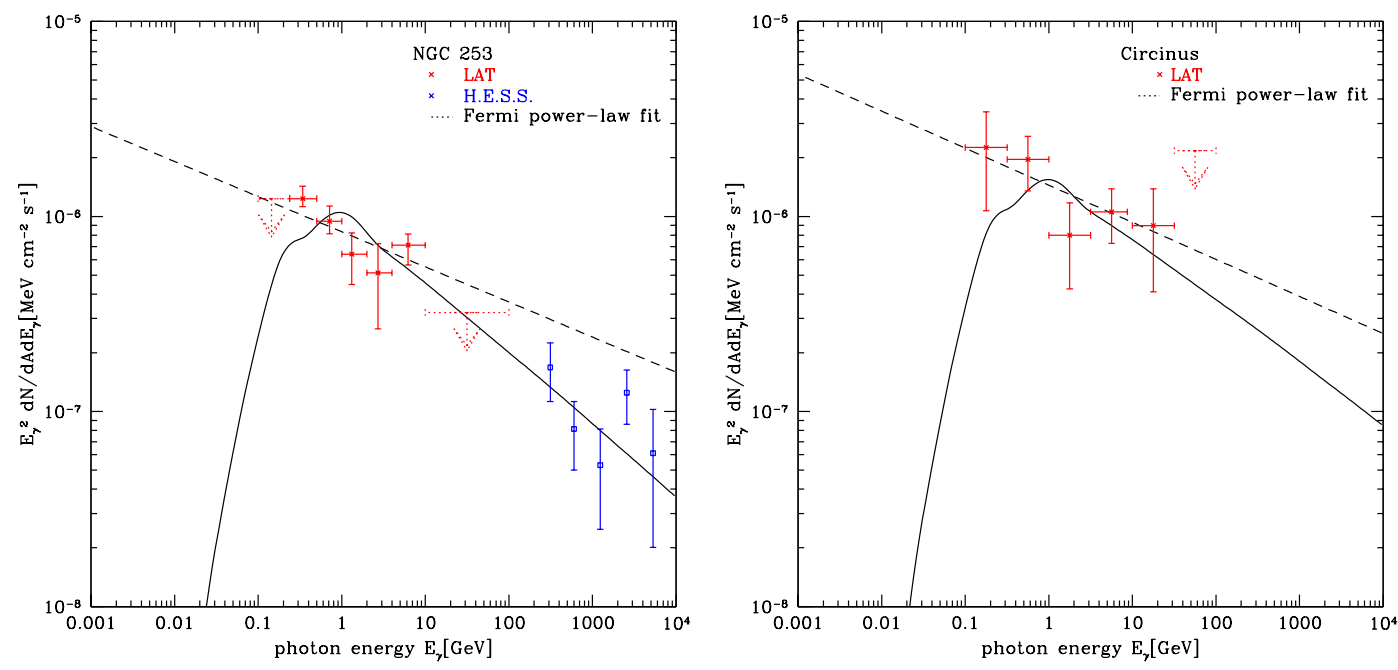

Figure 1: Left panel: Differential pionic gamma-ray spectrum (solid curve) for NGC253 with the best-fit parameters: source CR index $s$ and accelerated CR energy per $\mathrm{SN} \varepsilon_{\mathrm{cr}}$. Fermi points are stars (red), H.E.S.S points are squares (blue), and black dotted line is Fermi's power-law fit to GeV data. References see Table 1. Right panel: Similar spectrum for Circinus.

good and fairly well constrained thanks to the relatively large energy range. For Circinus, only $\mathrm{GeV}$ data is available and even our simple model is poorly constrained.

For all starbursts, $\chi^{2}$ contours appear in Figure 2. Both NGC253 and M82 have TeV data and good $\mathrm{GeV}$ data, and thus both $s$ and $\varepsilon_{\mathrm{cr}}$ are well-constrained. For these galaxies, we find $\varepsilon_{\mathrm{cr}} \sim 0.10$ foe, in good agreement with canonical estimates for Milky Way cosmic rays [2]. For NGC1068, NGC4945 and Circinus, lack of TeV data leaves the parameters poorly constrained, with large uncertainties in both $\varepsilon_{\mathrm{cr}}$ and $s$. For these galaxies, Table 1 thus reports the minimum $\varepsilon_{\mathrm{cr}}$ consistent with the data.

We adopt a maximum value of $\varepsilon_{\mathrm{cr}, \max }=0.3$ foe in order to judge the proton calorimetry of the starbursts: if $\varepsilon_{\mathrm{cr}}<\varepsilon_{\mathrm{cr}, \max }$, the starburst is a proton calorimeter with the calorimetric efficiency to be $\varepsilon_{\mathrm{cr}} / \varepsilon_{\mathrm{cr}, \max }$, i.e., M82 has a calorimetric efficiency of $35 \%$, NGC253 is $39 \%$, NGC1068 is $84 \%$ and NGC4945 is 70\%; if $\varepsilon_{\mathrm{cr}}>\varepsilon_{\mathrm{cr} \text {,max }}$, calorimetry fails for that galaxy as our model gives an upperlimit to the gamma-ray spectrum, other dominant gamma-ray sources must exist (e.g., AGNs) to explain the gamma-ray signal, therefore the Circinus galaxy is not a proton calorimeter. 

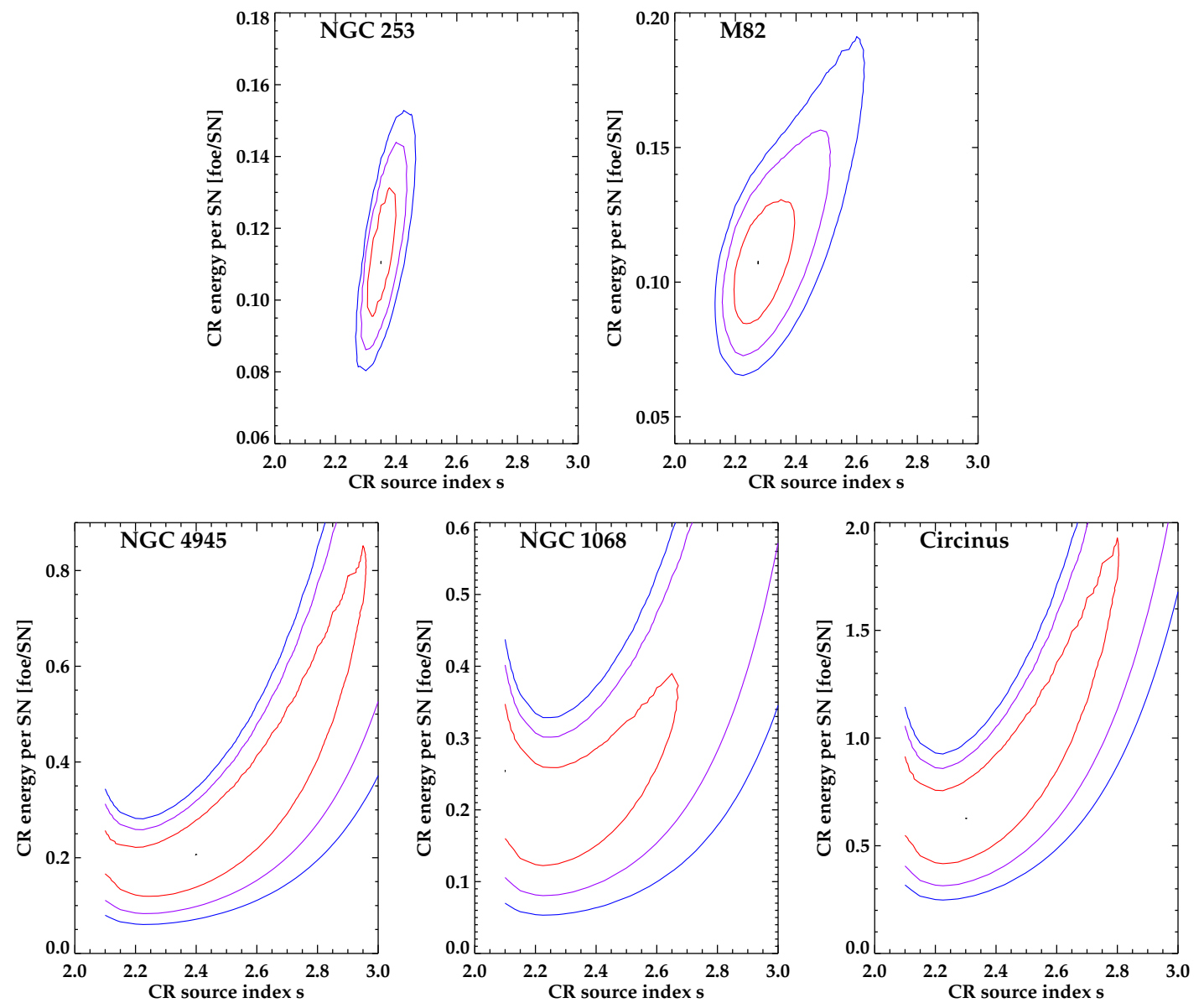

Figure 2: Contour plots for the $\chi^{2}$ test of five starburst galaxies: best-fit value is the black dot; (red, magenta, blue) lines represent (70\% CL, 95\% CL, 99\% CL). Above: Starbursts with well-constrained CR parameters $\mathrm{s}$ and $\varepsilon_{\mathrm{cr}}$ (has TeV data). Below: Starbursts with less-constrained CR s and $\varepsilon_{\mathrm{cr}}$ (lack of TeV data).

\subsection{Calorimetric Limit}

In a calorimeter case (again taken to be $\varepsilon_{\text {cr,max }}=0.3$ foe), eq. 2.2 gives $F_{\gamma} \propto R_{\mathrm{SN}} \propto \mathrm{SFR} \psi$, therefore $L_{\gamma} / \psi$ depends only on $s$. Using far-IR luminosity as a proxy for $\psi$, the expected calorimetric limit ratio $L_{>1 \mathrm{GeV}} / L_{8-100 \mu m}=5.2 \times 10^{-4}$ in our model with $s=2.0$. Ref. [22] calculated the ratio to be $3.1 \times 10^{-4}$ while Fermi group's result is $2.5 \times 10^{-4}$ [29].

The gamma-ray over far-IR luminosity ratio $L_{0.1-100 \mathrm{GeV}} / L_{8-100 \mu \mathrm{m}}$ expected in the calorimetric limit for CR nuclei is plotted in Fig 3. Our calorimetric limits agree with Fermi group's [29] result within 30\%, with different choices of the CR proton index $s$. Normal star-forming galaxies are about an order of magnitude below the calorimetric limits; starburst galaxies M82, NGC253, NGC1068 and NGC4945 are close to the limits, showing that good calorimetry relations hold for these galaxies, and starbursts have higher calorimetric efficiency than normal galaxies. The Circinus galaxy lies well above the limits, indicating other gamma-ray sources dominate. 


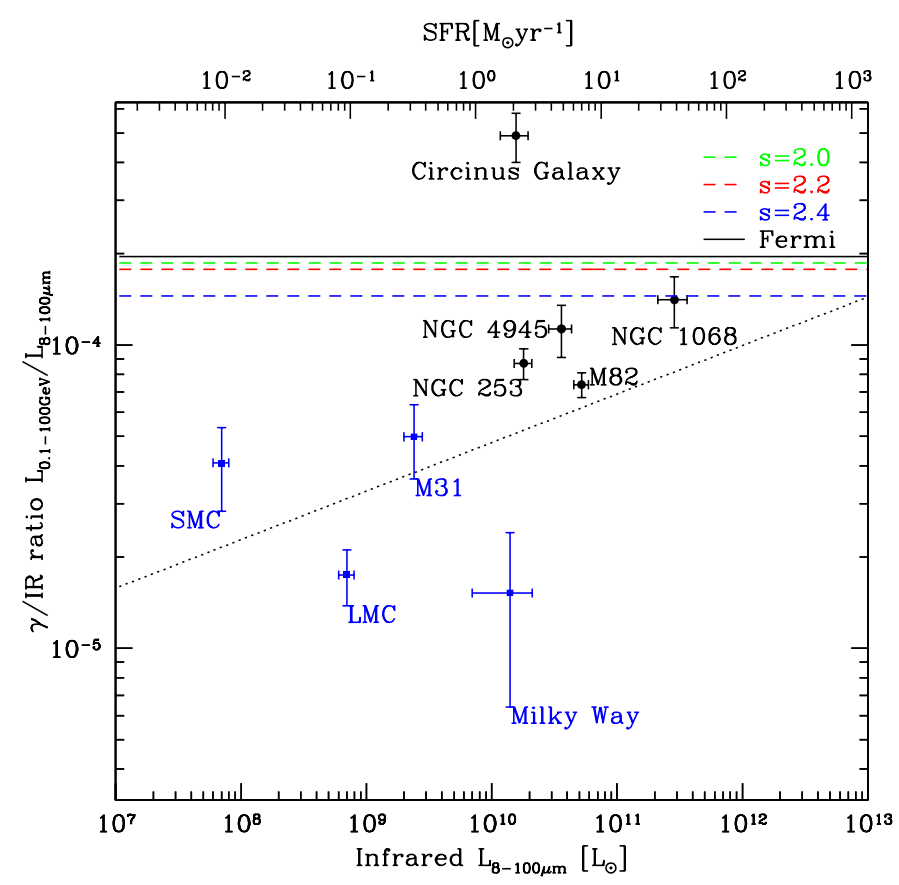

Figure 3: Plot of ratio of gamma-ray luminosity $(0.1-100 \mathrm{GeV})$ to total IR luminosity $(8-100 \mu \mathrm{m})$. Starforming galaxies are indicated with blue squares (IR and gamma-ray data of the Milky Way is calculated from [11], IR data for other galaxies is from [32], gamma-ray data for SMC is from [13], LMC is from [12], M31 is from [14]). Starburst galaxies are marked with black dots (IR data is from [33], gamma-ray data is from [29], except for Circinus [17]). The black dotted line is Fermi's best-fit power law relation [29]. The upper abscissa indicates SFR estimated from the IR luminosity according to the Kennicutt relation [34]. The colored dashed lines represents the expected gamma-ray luminosity in the calorimetric limit assuming an average $\mathrm{CR}$ acceleration energy per supernova of $\varepsilon_{\mathrm{cr}}=0.3$ foe with various source $\mathrm{CR}$ index $s=2.0,2.2,2.4$. The black solid line indicates Fermi's calorimetric result $\left(s=2.2, \varepsilon_{\mathrm{cr}}=0.1\right.$ foe) [29].

\section{Discussion}

In this paper, we construct a two-parameter, closed-box, thick-target model to test the cosmicray calorimetry in starburst galaxies. For the best-measured starbursts, our model gives good fits to the gamma-ray data in both $\mathrm{GeV}$ and $\mathrm{TeV}$ range with $s$ and $\varepsilon_{\text {cr }}$ values consistent with those of Galactic cosmic rays. The goodness of our fit to starbursts M82, NGC253, NGC1068 and NGC4945 suggests that starburst galaxies are proton calorimeters with various calorimetric efficiencies. These efficiencies may be different in reality if the actual supernova acceleration of CR rate in starbursts differs from the calorimeter value $\varepsilon_{\mathrm{cr} \text { max }}=0.3$ foe adopted. For the Circinus galaxy, our model's gamma-ray over-luminosity result agrees with ref. [17], indicating additional gamma-ray sources are needed to explain the observed data.

However, more data is needed to fill in the energy gap to do a further check of our model's results. There is a lack of data at energy $\sim 30-100 \mathrm{MeV}$ for the starburst galaxies, so it is difficult to tell whether the actual gamma-radiation has the characteristic "pion bump". TeV data for NGC1068, NGC4945 and Circinus is also needed to constrain the choices of parameters (both s and $\left.\varepsilon_{\mathrm{cr}}\right)$ in our model with smaller uncertainty. Moreover, recent NuStar X-ray limits on NGC253 
in the 7-20 $\mathrm{keV}$ band disfavors leptonic processes dominating in the $\mathrm{GeV}$ and $\mathrm{TeV}$ energy range [35].

\section{Acknowledgments}

We are pleased to thank Keith Bechtol for providing Fermi data, Wystan Benbow for VERITAS data. XW thanks the ICRC organizers for financial support, and for an enjoyable conference. This work was supported in part by the NASA ATP through award NNX10AC86G.

\section{References}

[1] W. Baade and F. Zwicky, Cosmic Rays from Super-novae, Proceedings of the National Academy of Science 20 (May, 1934) 259-263.

[2] V. L. Ginzburg and S. I. Syrovatskii, The Origin of Cosmic Rays. Macmillan, New York, 1964.

[3] F. Ackermann, M. et al., Detection of the Characteristic Pion-Decay Signature in Supernova Remnants, Science 339 (Feb., 2013) 807-811, [arXiv: 1302.3307].

[4] F. W. Stecker, Cosmic gamma rays, NASA Special Publication 249 (1971).

[5] C. D. Dermer, Secondary production of neutral pi-mesons and the diffuse galactic gamma radiation, A\&A 157 (Mar., 1986) 223-229.

[6] A. W. Strong, A. W. Wolfendale, and D. M. Worrall, Origin of the diffuse gamma ray background, MNRAS 175 (May, 1976) 23P-27P.

[7] G. G. Lichti, G. F. Bignami, and J. A. Paul, The gamma-ray luminosity of spiral galaxies - Its evolution and its contribution to the diffuse background above $100 \mathrm{MeV}$, ApSS 56 (July, 1978) 403-414.

[8] V. Pavlidou and B. D. Fields, Diffuse Gamma Rays from Local Group Galaxies, ApJ 558 (Sept., 2001) 63-71, [astro-ph/0105207].

[9] F. W. Stecker and T. M. Venters, Components of the Extragalactic Gamma-ray Background, ApJ 736 (July, 2011) 40, [arXiv:1012.3678].

[10] A. A. Abdo and Fermi LAT Collaboration, Fermi Large Area Telescope Measurements of the Diffuse Gamma-Ray Emission at Intermediate Galactic Latitudes, Physical Review Letters 103 (Dec., 2009) 251101, [arXiv:0912.0973].

[11] A. W. Strong and T. A. Porter, et al., Global Cosmic-ray-related Luminosity and Energy Budget of the Milky Way, ApJL 722 (Oct., 2010) L58-L63, [arXiv: 1008 . 4330].

[12] A. A. Abdo and M. Ackermann, et al., Observations of the Large Magellanic Cloud with Fermi, A\&A 512 (Mar., 2010) A7.

[13] A. A. Abdo and M. Ackermann, et al., Detection of the Small Magellanic Cloud in gamma-rays with Fermi/LAT, A\&A $\mathbf{5 2 3}$ (Nov., 2010) A46.

[14] A. A. Abdo and M. Ackermann, et al., Fermi Large Area Telescope observations of Local Group galaxies: detection of $M 31$ and search for M 33, A\&A 523 (Nov., 2010) L2, [arXiv:1012.1952].

[15] A. A. Abdo and Fermi LAT Collaboration, Detection of Gamma-Ray Emission from the Starburst Galaxies M82 and NGC 253 with the Large Area Telescope on Fermi, ApJL 709 (Feb., 2010) L152-L157, [arXiv:0911.5327]. 
[16] P. L. Nolan and A. A. Abdo, et al., Fermi Large Area Telescope Second Source Catalog, ApJS 199 (Apr., 2012) 31, [arXiv:1108.1435].

[17] M. Hayashida and Ł. Stawarz, et al., Discovery of GeV Emission from the Circinus Galaxy with the Fermi Large Area Telescope, ApJ 779 (Dec., 2013) 131, [arXiv: 1310.1913$].$

[18] V. A. Acciari and VERITAS Collaboration, A connection between star formation activity and cosmic rays in the starburst galaxy M82, Nature 462 (Dec., 2009) 770-772, [arXiv: 0911.0873 ].

[19] F. Acero and F. Aharonian, et al., Detection of Gamma Rays from a Starburst Galaxy, Science 326 (Nov., 2009) 1080-, [arXiv:0909.4651].

[20] A. Abramowski and H. E. S. S. Collaboration, Spectral Analysis and Interpretation of the $\gamma$-Ray Emission from the Starburst Galaxy NGC 253, ApJ 757 (Oct., 2012) 158, [arXiv: 1205.5485 ].

[21] M. Pohl, On the predictive power of the minimum energy condition. I - Isotropic steady-state configurations, A\&A 270 (Mar., 1993) 91-101.

[22] B. C. Lacki and T. A. Thompson, et al., On the GeV and TeV Detections of the Starburst Galaxies M82 and NGC 253, ApJ 734 (June, 2011) 107, [arXiv: 1003.3257 ].

[23] M. Persic, Y. Rephaeli, and Y. Arieli, Very-high-energy emission from M 82, A\&A 486 (July, 2008) 143-149.

[24] E. de Cea del Pozo, D. F. Torres, and A. Y. Rodriguez Marrero, Multimessenger Model for the Starburst Galaxy M82, ApJ 698 (June, 2009) 1054-1060, [arXiv: 0901 . 2688].

[25] T. M. Yoast-Hull and J. E. Everett, et al., Winds, Clumps, and Interacting Cosmic Rays in M82, ApJ 768 (May, 2013) 53, [arXiv: 1303.4305$].$

[26] X. Wang and B. D. Fields, Are Startburst Galaxies Proton Calorimeters? in preparation, 2015, .

[27] M. Meneguzzi, J. Audouze, and H. Reeves, The production of the elements Li, Be, B by galactic cosmic rays in space and its relation with stellar observations., A\&A 15 (1971) 337-359.

[28] R. J. Gould, Effects of nuclear forces on ion thermalization in high-temperature plasmas, ApJ 263 (Dec., 1982) 879-890.

[29] M. Ackermann and M. Ajello, et al., GeV Observations of Star-forming Galaxies with the Fermi Large Area Telescope, ApJ 755 (Aug., 2012) 164, [arXiv:1206.1346].

[30] T. A. D. Paglione and R. D. Abrahams, Properties of nearby Starburst Galaxies Based on their Diffuse Gamma-Ray Emission, ApJ 755 (Aug., 2012) 106, [arXiv: 1206.3530 ].

[31] F. Aharonian and A. G. Akhperjanian, et al., Observations of selected AGN with HESS, A\&A 441 (Oct., 2005) 465-472, [astro-ph/0507207].

[32] D. B. Sanders and J. M. Mazzarella, et al., The IRAS Revised Bright Galaxy Sample, The Astronomical Journal 126 (Oct., 2003) 1607-1664, [astro-ph / 0306263 ].

[33] Y. Gao and P. M. Solomon, The Star Formation Rate and Dense Molecular Gas in Galaxies, ApJ 606 (May, 2004) 271-290, [astro-ph/0310339].

[34] R. C. Kennicutt, Jr., The Global Schmidt Law in Star-forming Galaxies, ApJ 498 (May, 1998) 541-552, [astro-ph/9712213].

[35] D. R. Wik and B. D. Lehmer, et al., Spatially Resolving a Starburst Galaxy at Hard X-Ray Energies: NuSTAR, Chandra, and VLBA Observations of NGC 253, ApJ 797 (Dec., 2014) 79, [arXiv:1411.1089]. 